ORIGINAL ARTICLE

\title{
Does radio-opaque contrast improve radiographic localisation of percutaneous central venous lines?
}

\author{
D E Odd, B Page, M R Battin, J E Harding
}

See end of article for authors' affiliations

Correspondence to:

Professor J Harding,

Newborn Services,

National Women's

Hospital, Private Bag

92189, Auckland, New

Zealand;

i.harding@auckland.ac.nz

Accepted

20 November 2002
Background: Percutaneous central venous lines (long lines) are commonly used in neonatal practice. The position of these lines is important, because incorrect placement may be associated with complications. Aims: To determine whether the addition of radio-opaque contrast material improves the localisation of long line tips over plain radiography.

Methods: Radiographs taken to identify long line position were identified in two periods; 106 radiographs without contrast taken between October 1999 and August 2000, and 96 radiographs with contrast between September 2001 and July 2002. Two observers independently reviewed each radiograph to identify the position of the line tip. The formal radiology report was recorded as a third observer.

Results: The use of contrast increased the proportion of radiographs in which all observers reported they could see the long line tip $(53(55 \%)$ v $41(39 \%))$. It also increased the proportion where they agreed on anatomical position $(57(59 \%) v 39(37 \%))$ and there was a higher kappa coefficient for agreement $(0.56 v$ 0.33).

Conclusions: The use of contrast while taking radiographs for the localisation of long line position improves the likelihood that an observer can see a long line tip and reduces inter-observer variability. Even using contrast, precise localisation of a long line tip can be difficult.
$\mathrm{P}$ ercutaneous central venous lines (long lines) are commonly used in neonatal practice, where central venous access is often necessary for weeks to allow parenteral feeding. The position of these lines is important, because incorrect placement may be associated with complications, including cardiac tamponade, ${ }^{12}$ thrombosis, and sepsis. $^{34}$

The UK Department of Health released guidelines in June 2001 after the deaths of four babies in England were linked to long line use and central placement of the long line tips. ${ }^{1}$ Recommendations were made to place the tip outside the cardiac chambers. However, the best way to identify the position of these lines is unclear. The Department of Health recommended the use of plain radiography, ${ }^{1}$ while others have advocated the routine use of intravenous contrast material to clarify the position. ${ }^{5}$

The aim of this study was to determine whether the addition of contrast material improved the localisation of long line tips on radiographs. As there is widespread variability in interpretation of radiographs, ${ }^{68}$ we also assessed whether the addition of contrast material reduced inter-observer variability in reporting of long line position.

\section{METHODS}

A retrospective study was conducted at a regional neonatal intensive care unit. All infants who had long lines inserted from October 1999 to August 2000 were prospectively identified in an ongoing audit during this time. Plain radiographs without contrast were taken to identify the long line position during this period.

In August 2001, unit practice changed to include the use of radio-opaque contrast to localise long line tips. All infants admitted to the newborn unit from September 2001 to July 2002 who had radiographs with contrast to localise a long line tip were identified from the radiology department records. Radiographs and the corresponding formal radiologist's report were obtained for review. Only the first radiograph for each long line inserted was included.
After insertion of each long line a radiograph was taken and reviewed prior to the line being used to infuse fluids. The recommended position for long lines was with the tip placed just outside the cardiac chambers, and sites for insertion were the long saphenous vein, posterior tibial vein, or a large vein in the antecubital fossa. Practitioners were advised to measure from the site of insertion to the xiphisternum and to initially insert the catheter this distance. Because an internal audit found that a small number of long lines inserted via the arms were being placed initially into the cardiac chambers, from February 2002 the guideline was modified, stating that for long lines placed in the arms the measurement should be to the sternal notch. However, this did not significantly alter the reported placement of lines in this study, perhaps because the change occurred late in the study period and thus the numbers of potentially affected lines were small. Therefore data from before and after this change in guideline have been combined in this report.

Catheters used were silicone 23 gauge Epicutaneo-CavaKatheter (Vygon Corp., East Rutherford, NJ, United States) throughout this study. Iohexol $302 \mathrm{mg} / \mathrm{ml}$ (Omnipaque 140, Nycomed NZ Ltd, Auckland, NZ) was the contrast medium used. A $0.5 \mathrm{ml}$ aliquot of $0.9 \%$ saline was instilled into the line to check patency, followed by $0.5 \mathrm{ml}$ of iohexol. The radiograph was taken and the line flushed again with $0.5 \mathrm{ml}$ of $0.9 \%$ saline. Contrast material was not injected while the radiograph was being taken.

A neonatal fellow and a neonatal nurse practitioner independently reviewed each radiograph to identify the position of the line tip. They recorded whether the tip could be identified (yes, maybe, or no) and its position. If the observer was unable to see the tip the most likely position was recorded. The formal radiology report issued routinely, independent of this study, was recorded as a third observer. Certainty on line tip position was assumed unless the report qualified the location with the word "likely", "possibly", "maybe" or "probably", or it was specified that the position could not be identified. The location was considered 
Table 1 Comparison between the study groups

\begin{tabular}{lll}
\hline & No contrast & Contrast \\
\hline Radiographs reviewed & 106 & 96 \\
Number of babies & 92 & 89 \\
Males (n) & $58(54 \%)$ & $59(61 \%)$ \\
Gestation (weeks) & $28(23-40)$ & $28(23-41)$ \\
Birth weight (grams) & $1190(540-3100)$ & $1110(475-4380)$ \\
Age at insertion (days) & $2(0-49)$ & $2(0-84)$ \\
Lines inserted via lower limb & $41(38 \%)$ & $35(37 \%)$ \\
Babies with multiple lines* & $13(14 \%)$ & $6(7 \%)$ \\
\hline
\end{tabular}

Values are number (\%) or median (range) as appropriate. There were no significant differences between the groups.

*One baby in each group had three lines inserted.

unsatisfactory if it was within the cardiac chambers or in an aberrant vessel.

Inter-observer variability was assessed by proportion of agreement and by using Cohen's kappa coefficient as a measure of chance corrected agreement. ${ }^{9}$ Continuous variables were analysed by the Mann-Whitney $U$ test and categorical variables were analysed by the $\chi^{2}$ test using Statview (SAS Institute, Inc., Cary, NC, USA). Values are expressed as median (range) or number (\%) as appropriate.

\section{RESULTS}

\section{Study population}

A total of 119 radiographs without contrast and 100 radiographs with contrast were identified. In 13 of the radiographs without contrast and four of those with contrast, the films could not be obtained or the formal radiology report did not comment on the long line position. This left 106 radiographs from 92 infants in the non-contrast group and 96 radiographs from 89 infants in the contrast group, which were included in this study. Median gestations were 28 (23-40) and 28 (2341) weeks, and birth weights were $1190 \mathrm{~g}$ (540-3100 g) and $1110 \mathrm{~g}(475-4380 \mathrm{~g})$ in the non-contrast and contrast group respectively. One third of the lines were inserted via the lower limbs in each group (41 non-contrast, 35 contrast) (table $\left.\mathrm{I}^{(\mathrm{T} 1)}\right)$. In each case, three reports were obtained for each radiograph, one from each observer.

\section{Line position}

The reported position of the line tip was different between the two groups $(p=0.0007)$, with the non-contrast group having more lines reported in the ventricle (21 (7\%) v $6(2 \%)$ reports) and fewer in the distal veins $(50$ (16\%) v 68 (24\%) reports) (table 2(T2)). The most frequently reported position was in the superior or inferior vena cava in both groups (30$36 \%$ non-contrast, $30-40 \%$ contrast) (table $2^{(\mathrm{T} 2)}$ ).

Table 2 Comparison between reports of lines with or without contrast

\begin{tabular}{lll}
\hline & No contrast & Contrast \\
\hline $\begin{array}{l}\text { Number of reports } \\
\text { Positions reported }\end{array}$ & 318 & 288 \\
Distal vein & $50(13-19 \%)$ & $68(22-25 \%)$ \\
lliac & $25(6-11 \%)$ & $33(9-14 \%)$ \\
Superior or inferior vena cava & $105(30-36 \%)$ & $103(30-40 \%)$ \\
Atrium & $80(22-30 \%)$ & $62(19-24 \%)$ \\
Ventricle & $21(4-11 \%)$ & $6(0-4 \%)$ \\
Other & $37(7-17 \%)$ & $16(2-7 \%)$ \\
\hline
\end{tabular}

Values are number of reports (range of reported percentages). Three reports (one from each observer) were obtained for each radiograph. Reported positions are different between non-contrast and contrast groups $(p=0.0002)$.

\section{Inter-observer variation}

The three observers reported the long line tip as seen in 66$79 \%$, maybe seen in $20-29 \%$, and not seen in $1-5 \%$ of the radiographs. The observers thought the tip was in a satisfactory position in $85-88 \%$ of radiographs.

The observers all agreed that they saw the line tip in $47 \%$ of radiographs and agreed on position in $48 \%$ of radiographs. Agreement on the position was not affected by gender, gestation, birth weight of the baby, or age at insertion of the line. Agreement was more likely if the line was inserted via the lower limb as opposed to the upper limb or scalp (43 $(57 \%) \vee 53(42 \%), \mathrm{p}=0.05)$.

\section{Effect of contrast}

The use of contrast increased the proportion of radiographs in which all three observers reported they could see the long line tip $(53(55 \%) \vee 41 \quad(39 \%), p=0.02)$. It also increased the proportion where they agreed on anatomical position (57 $(59 \%) \vee 39(37 \%), p=0.001)$ and there was a higher kappa coefficient for agreement $(0.56 v 0.33)$. The use of contrast improved the proportion of radiographs in which all three observers reported a satisfactory placement (84 (88\%) $v 81$ $(76 \%), p=0.04)$, although there was no improvement in the kappa coefficient for agreement $(0.47 v 0.41)$.

In the subgroup of radiographs where one or more observers could not see the long line tip, the use of contrast improved the proportion in which the observers agreed on position $(24(56 \%) \vee 13(20 \%), p=0.0001)$. However, when all three observers felt they could see the tip, use of contrast did not improve the proportion in which they agreed (33 $(62 \%) \vee 26(63 \%), \mathrm{p}=0.91)$.

\section{DISCUSSION}

Localisation of the tip of a long line is important in order to reduce the chance of adverse effects as a result of inappropriate line placement. ${ }^{1-4}$ We found that the use of contrast improves the localisation of long lines on radiography, but that even when contrast is used, precise localisation can be difficult.

The use of contrast improved the proportion of line tips seen by all three observers from one third to over a half. It also improved the proportion of lines in which the observers all agreed on the anatomical position by a similar amount. The improvement in proportion of agreement was confined to those long lines where one or more of the observers could not see the line tip. Where they all saw the line tip, contrast did not improve the inter-observer variability, and agreement between the two groups was similar. This suggests that contrast helps the localisation of line tips only in the radiographs that are more difficult to assess.

The kappa coefficient in this study has been used as a way to quantify the level of agreement, while correcting for chance. A kappa value of one would imply complete agreement of the three observers, a value of zero would suggest there was no agreement other than that which would be expected by chance, and a value of minus one would imply complete disagreement. Interpretation of kappa has been suggested $^{10}$ to consider levels between 0.21 and 0.40 consistent with poor, between 0.41 and 0.60 consistent with moderate, and above 0.60 consistent with good agreement. Using these criteria there was an improvement from poor (0.33) to moderate $(0.56)$ agreement on position with the use of contrast.

Inter-observer variation must be taken into account in the interpretation of any radiographic investigation. Overall agreement in this study was poor. There was agreement on position in only $48 \%$ of radiographs, and even when the three observers all reported they could identify the long line tip there was agreement on position in only $63 \%$. However, the 
kappa values in our study do not differ greatly from values reported in other studies looking at inter-observer variation on radiography for the diagnosis of other pathologies. ${ }^{68}$

There was a difference in reported position of the long line tip between the two study groups, with the tip more likely to be reported in the ventricle and less likely in a distal vein in the non-contrast group. The reason for this is unclear. The use of contrast itself may affect the position reported by making the tip easier to see. In particular, the reporting of centrally placed lines could be affected, where the mediastinal shadow may obscure the line position in the noncontrast group. However, it seems unlikely that the change in position itself could account for the improved inter-observer variability since the majority of the lines (57-58\%) were reported to lie in the vena cavae or atria in both groups.

We also found that long lines inserted via the legs appear to be better visualised than those inserted via the arms or scalp. This may be because of the lack of radio-opaque structures in the abdomen compared to the mediastinum. However, this also does not account for any change in reported position with contrast, since the proportion of lines inserted via the legs was similar in both groups.

Only one previous study ${ }^{5}$ has examined the use of contrast to localise long line position in neonates. In that study, plain radiography without contrast was used initially, and if the tip was not visible to a single observer, then a contrast radiograph was also taken. Half the line tips could not be visualised without contrast, but nearly all could be seen with the second radiograph. Recommendations were made for the routine use of contrast to determine long line position. Our study took a different approach, with infants only having a single radiograph and hence avoiding the potential confounding factor of multiple radiographs in only one arm of the study. In addition, we used a large number of radiographs and multiple observers to determine the position of the long line tip to reduce the subjective nature of its localisation.

Alternative techniques to improve localisation of long lines have been suggested. Ultrasound ${ }^{11}{ }^{12}$ has been shown to be effective but is operator dependent. The use of digital imaging $^{13}$ has been suggested, having the advantage that the resulting image can be manipulated on the computer screen in order the make the long line tip more conspicuous. Long lines containing metallic guidewires ${ }^{14}$ have also been advocated, although they have not been compared with plain radiography.

When contrast is used in our unit, each infant receives $0.5 \mathrm{ml}$ of iohexol $(302 \mathrm{mg} / \mathrm{ml})$ to identify the long line. The concentration of iodine was $140 \mathrm{mg} / \mathrm{ml}$ in the preparation used, making the mean dose of iodine given to the infants in our study population $55 \mathrm{mg} / \mathrm{kg}$. This is much lower than the lowest recommended dose from the manufacturers (Nycomed NZ Ltd, Auckland, NZ) for intravenous use $(300 \mathrm{mg} / \mathrm{kg}$ ), but could not be considered insignificant. Adverse effects of iodinated contrast reported in adults include platelet dysfunction, ${ }^{15}$ anticoagulant effects, ${ }^{16}$ and renal impairment. ${ }^{17}$ We did not look at adverse effects of contrast in this study and the effects of iodinated contrast material are relatively unknown in neonates. However, some studies have reported that the dose of iodine received in contrast material has been large enough to affect thyroid function. ${ }^{18} 19$ Other theoretical risks include the risk that repeated exposure to contrast may increase the likelihood of allergic reactions in later life, and the increased risk of sepsis as a result of the need to break into the line to administer the contrast. These potential risks need to be taken into account when considering the use of contrast in clinical practice.

The use of radio-opaque contrast while taking radiographs for the localisation of long line position improves the likelihood that an observer can see the tip and reduces the inter-observer variability. When using radiology to identify long line tip in neonates we recommend the routine use of contrast. However, even with the use of contrast, precise localisation of a long line tip can be difficult.

\section{Authors' affiliations}

D E Odd, B Page, M R Battin, J E Harding, Newborn Services, National Women's Hospital, Private Bag 92189, Auckland, New Zealand

\section{REFERENCES}

1 DoH. Review of four neonatal deaths due to cardiac tamponade associated with the presence of a central venous catheter. Recommendations and Department of Health response.http://www.doh.gov.uk/manchesterbabies/ manchestersummary.

2 Beattie PG, Kuschel CA, Harding JE. Pericardial effusion complicating a percutaneous central venous line in a neonate. Acta Paediatr 1993;82:105-7.

3 Neubauer AP. Percutaneous central iv access in the neonate: experience with 535 silastic catheters. Acta Paediatr 1995;84:756-60.

4 Racadio JM, Doellman DA, Johnson ND, et al. Pediatric peripherally inserted central catheters: complication rates related to catheter tip location. Pediatrics 2001; 107:E28

5 Reece A, Ubhi T, Craig AR, et al. Positioning long lines: contrast versus plain radiography. Arch Dis Child Fetal Neonatal Ed 2001;84:F129-30.

6 Bloomfield FH, Teele RL, Voss M, et al. Inter- and intra-observer variability in the assessment of atelectasis and consolidation in neonatal chest radiographs. Pediatr Radiol 1999;29:459-62.

7 Melbye H, Dale K. Interobserver variability in the radiographic diagnosis of adult outpatient pneumonia. Acta Radiol 1992;33:79-81.

8 Collins CD, Wells $\mathrm{AU}$, Hansell DM, et al. Observer variation in pattern type and extent of disease in fibrosing alveolitis on thin section computed
tomography and chest radiography. Clin Radiol 1994;49:236-40.

9 Cohen J. A coefficient of agreement for nominal scales. Educational and Psychological Measurement 1960;20:37-46.

10 Brennan P, Silman A. Statistical methods for assessing observer variability in clinical measures. BMJ 1992;304:1491-4.

11 Diemer A. Central venous silastic catheters in newborns: localization by sonography and radiology. Pediatr Radiol 1987;17:15-17.

12 Madar RJ, Deshpande SA. Reappraisal of ultrasound imaging of neonatal intravascular catheters. Arch Dis Child Fetal Neonatal Ed 1996;75:F62-4.

13 Bernard I, Banerjee I. Image inversion [e-letter]. Arch Dis Child Fetal Neonatal Ed http://adc.bmijournals.com/cgi/eletters/fetalneonatal;84/2/F129\#75 (posted 13 June 2001).

14 Yadav M. Where should the long line tip lie? [e-letter]. Arch Dis Child Fetal Neonatal Ed http://adc.bmijournals.com/cgi/eletters/fetalneonatal;84/2/ F129\#71 (posted 14 May 2001).

15 Hardeman MR, Konijnenberg A, Sturk A, et al. Activation of platelets by lowosmolar contrast media: differential effects of ionic and nonionic agents. Radiology 1994; 192:563-6.

16 Rasuli P, McLeish WA, Hammond DI. Anticoagulant effects of contrast materials: in vitro study of iohexol, ioxaglate, and diatrizoate. AJR Am J Roentgenol 1989;152:309-11.

17 Lasser EC, Lyon SG, Berry CC. Reports on contrast media reactions: analysis of data from reports to the U.S.Food and Drug Administration. Radiology 1997;203:605-10.

18 Parravicini E, Fontana C, Paterlini GL, et al. lodine, thyroid function, and very low birth weight infants. Pediatrics 1996;98:730-4.

19 I'Allemand D, Grüter A, Beyer P, et al. lodine in contrast agents and skin disinfectants is the major cause for hypothyroidism in premature infants during intensive care. Horm Res 1987;28:42-9. 